\title{
A comparative study of the cooling-rate effect on rock strength reduction after microwave irradiation
}

\author{
Hamed Rafezi ${ }^{1, *}$ (D), Samir M. Deyab ${ }^{1}$, Ferri Hassani ${ }^{1}$ and Seyed Ali Ghoreishi-Madiseh ${ }^{2}$ \\ ${ }^{1}$ Department of Mining and Materials Engineering, McGill University, Montreal, QC H3A 2A7, Canada, and ${ }^{2}$ Norman B. Keevil \\ Institute of Mining Engineering, University of British Columbia, Vancouver, BC V6T 1Z4, Canada \\ ${ }^{*}$ Corresponding author: hamed.rafezi@mcgill.ca
}

(Received 26 June 2020; Revised 09 July 2020; Accepted 10 July 2020)

\begin{abstract}
A variety of machines are currently being used for mechanical excavation in mining and civil industries. A series of research works have been conducted at McGill University in the past decade to study the effects of microwave (MW) irradiation on rock mechanical properties. The idea is to enhance the excavation performance by improving the rate of penetration and decreasing the wear rate on the cutting tools. These two effects would eventually translate into economic benefits for mine operators. The effectiveness of MW on weakening rocks is proven, however the most efficient method to employ MW in mines is still under investigation. This article presents some experimental results on the effects of cooling- rate on rock strength. Brazilian Tensile Strength (BTS) of microwave treated samples were compared in natural air-cooled and water rapid-cooled conditions.
\end{abstract}

Keywords: Mining; Microwave irradiation; Rock preconditioning; Rock excavation

\section{Objective}

To determine the impact of rapid cooling of rock samples after MW treatment and compare the mechanical properties with the air-cooled condition.

\section{Introduction}

Roadheaders are usually used for rock with an unconfined compressive strength value of less than $150 \mathrm{MPa}$. In tunnel boring machines (TMBs), a high wear rate is another factor that challenges the economic feasibility of the technology. MW irradiation for rock strength reduction prior to the excavation was proposed by Maurer, 1968. He used MW at a maximum of $4.8 \mathrm{~kW}$ to initiate cracks within the rock samples and reduce the required mechanical force for excavation. Based on this concept, researchers have been working on the application of MW in a variety of mechanical cutting processes (Hassani \& Nekoovaght, 2011; Jerby et al., 2002; 2018; Lindroth et al., 1991). Ongoing researches are attempting to optimize the idea of MW rock preconditioning in physical and economical aspects. The present article is assessing the impact of rapid cooling as compared to air cooling on the rock strength after MW irradiation. Comprehensive research work is currently undergoing in collaboration with the University of British Columbia to analyze different aspects of MW application for rock preconditioning; from numerical simulation to experimental validation and energy analysis. 


\section{Method}

Basalt rock samples were cut into standard disk size for BTS testing (i.e. diameter of $50 \mathrm{~mm}$ and thickness of $25 \mathrm{~mm}$ ). A total of fifteen samples were tested under three different test conditions; namely, nontreated, MW treated and rapid-cooled, and MW treated and air-cooled. Five samples were dedicated to each test group. All the treated samples were exposed to MW irradiation for 5 seconds at $15 \mathrm{~kW}$ of power using the MW system shown in figure 1 . For air cooling, the samples were cooled in room temperature for 20 minutes, while the rapid-cooled samples were placed into room temperature $\left(22^{\circ} \mathrm{C}\right)$ water container for 2 minutes prior to the BTS measurement. Cooling times were defined based on the experimental observations that air-cooling was found to be an order of magnitude slower than water-cooling; i.e. approximately 20 minutes for air-cooling and 2 minutes for water-cooling.

\section{Results and Discussion}

The aim of this study was to assess the cooling condition on the effect of MW treatment on basalt rock samples. The treated samples were exposed to MW irradiation at the same power and for the same duration. As plotted in figure 2, the average BTS for non-treated basalt samples was found to be

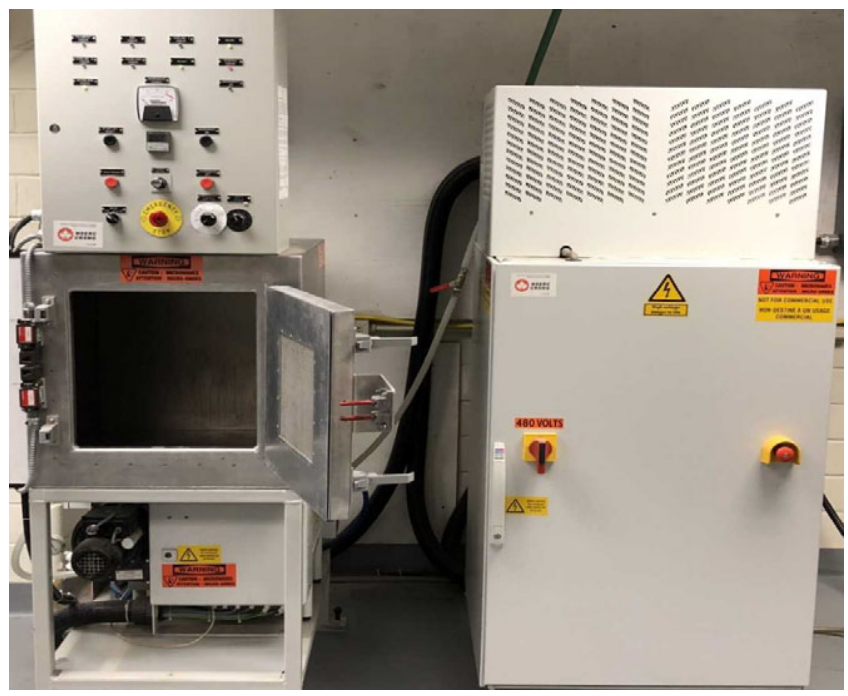

Fig. 1 Industrial microwave system (Thermex Thermatron, 15 kW, $2.4 \mathrm{GHz}$ ).

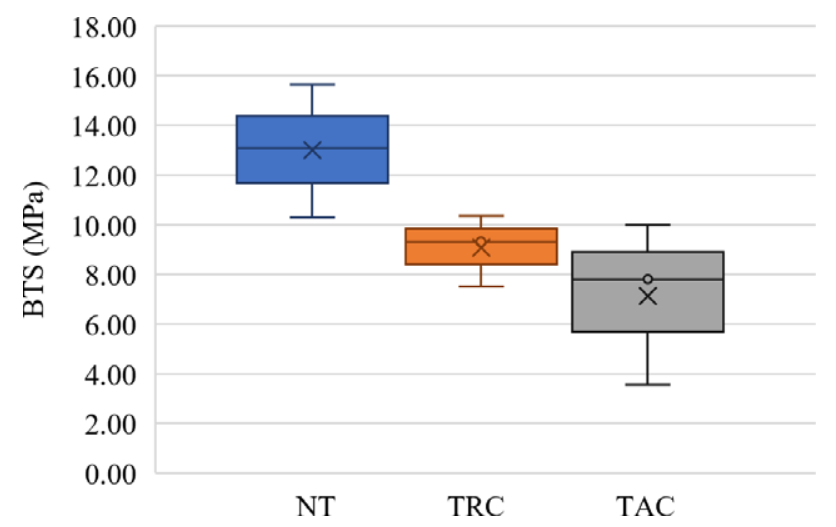

Fig. 2 Brazilian tensile strength (BTS) test results for three test conditions; NT: non-treated, TRC: treated (rapid-cooled) and TAC: treated (air-cooled). 
13.01 MPa while this value for rapid-cooled and air-cooled was measured as 9.07 $\mathrm{MPa}$ and 7.12 $\mathrm{MPa}$, respectively. These results show that the prolonged low-temperature cooling process in basalt samples works in favor of rock weakening and improves the results at the same level of MW application.

\section{Conclusions}

This short article presents the experimental outcomes of a series of tests to compare the microwave treatment effectiveness in rock weakening at different cooling conditions. The results show that the aircooled basalt rock samples experienced about $45 \%$ reduction in BTS values while this change was found to be about $30 \%$ for rapid-cooled samples. These results confirm the improvement in the weakening procedure of basalt rock in terms of BTS in air-cooled condition. The behavior of other rock types depending on their mineral composition and grain configuration are yet to be studied.

Funding. This work was supported by Natural Sciences and Engineering Research Council of Canada NSERC (Discovery grant \# 03715, CRD grant\#05395) as well as DeBeers, Metso and Arjex companies.

Conflicts of Interest Declaration. Authors declare none.

Data Availability. The data are enclosed in the paper.

\section{References}

Hassani, F., \& Nekoovaght, P. (2011). The development of microwave assisted machineries to break hard rocks. 2011 Proceedings of the 28th ISARC, Seoul, Korea (pp. 678-684). International Association for Automation and Robotics in Construction (IAARC). https://doi.org/10.22260/ISARC2011/0127

Jerby, E., Dikhtyar, V., Aktushev, O., \& Grosglick, U. (2002). The microwave drill. Science, 298, 587-589. https://doi.org/ 10.1126/science.1077062.

Jerby, E., Nerovny, Y., Meir, Y., Korin, O., Peleg, R., \& Shamir, Y. (2018). A silent microwave drill for deep holes in concrete. IEEE Transactions on Microwave Theory and Techniques, 66, 522-529. https://doi.org/10.1109/TMTT.2017.2729509.

Lindroth, D. P., Morrell, R. J., \& Blair, J. R. (1991). Microwave assisted hard rock cutting. U.S. Patent No. 5,003, 144. U.S. Patent and Trademark Office.

Maurer, W. C. (1968). Novel drilling techniques. Pergamon Press.

Cite this article: Rafezi H, Deyab SM, Hassani F, Ghoreishi-Madiseh SA (2020). A comparative study of the cooling-rate effect on rock strength reduction after microwave irradiation Experimental Results, 1, e36, 1-6. https://doi.org/10.1017/exp.2020.32 


\section{Peer Reviews}

\section{Reviewing editor: Dr. Daniel Micallef}

University of Malta, Environmental Design, Tal-Qroqq,, Msida, South, Malta, MSD2080

This article has been accepted because it is deemed to be scientifically sound, has the correct controls, has appropriate methodology and is statistically valid, and met required revisions.

doi:10.1017/exp.2020.32.pr1

\section{Review 1: Effect of rapid cooling on rock strength reduction after microwave irradiation}

Reviewer: Dr. Mohammed Hefni

Date of review: 29 June 2020

(c) The Author(s), 2020. Published by Cambridge University Press This is an Open Access article, distributed under the terms of the Creative Commons Attribution licence (http://creativecommons.org/licenses/by/4.0/), which permits unrestricted re-use, distribution, and reproduction in any medium, provided the original work is properly cited.

Conflict of interest statement. "Reviewer declares none"

Comments to the Author: A design of experiment approach may be helpful in this kind of study and you can consider using it in future publications. I also recommend conducting an economic analysis to investigate the viability of this method in the future. otherwise, the manuscript is well written

\section{Score Card}

Presentation

4.0

Is the article written in clear and proper English? (30\%)

Is the data presented in the most useful manner? (40\%)

Does the paper cite relevant and related articles appropriately? (30\%)

Context

Does the title suitably represent the article? (25\%)

Does the abstract correctly embody the content of the article? (25\%)

Does the introduction give appropriate context? (25\%)

Is the objective of the experiment clearly defined? (25\%)

Are the limitations of the experiment as well as the contributions of the

experiment clearly outlined? $(20 \%)$ 


\title{
Review 2: Effect of rapid cooling on rock strength reduction after microwave irradiation
}

\author{
Reviewer: Dr. Eli Jerby (1)
}

Date of review: 07 July 2020

\begin{abstract}
(C) The Author(s), 2020. Published by Cambridge University Press This is an Open Access article, distributed under the terms of the Creative Commons Attribution licence (http://creativecommons.org/licenses/by/4.0/), which permits unrestricted re-use, distribution, and reproduction in any medium, provided the original work is properly cited.
\end{abstract}

Conflict of interest statement. Reviewer declares none.

Comments to the Author: This concise paper presents an interesting contribution to the paradigmatic framework of microwave impacts on rocks. While most other studies deal with the microwave-heating process as a means for rock weakening (e.g. for mining or construction applications), this work investigates the impact of the (post-microwave) cooling rate on the final reduction of the rock strength. The main result of this study may seem counter-intuitive and even surprising. The authors report that the rapid-cooling (wet) process is found less effective (in terms of the rock weakening level achieved) than the natural air-cooling. This experimental result may influence other studies in the field, and possibly affect the design of future microwave-assisted machinery.

The following minor comments are proposed for the authors' consideration, in order to advance the paper accessibility by both experts in the field and the general readers of Experimental Results:

1. Title: Instead of "Effect of rapid cooling on..." please consider "A comparative study of the coolingrate effect on..." or "On the cooling-rate effect...", or similar.

2. Abstract: The abstract shall present a brief summary of the paper itself, its scientific content and significance. The institutional and collaborative aspects could be noted elsewhere in the paper. The general introductory sentences should be more clearly related to the present study. The last sentence in the abstract, which is fine, should be detailed and extended.

3. Keywords: Please consider adding the keyword "Microwave drilling" in order to combine mining and microwave aspects.

4. Objective, Line 24: Please consider "To determine the impact of..." instead of "To determine the effect of..."

5. Figure 1: Please consider the caption "The microwave experimental setup based on a $15-\mathrm{kW}$ industrial system". Please provide its brand and model number in the text.

6. Figure 2: This figure may self-consistently standalone (as a graphical abstract, etc.) hence the abbreviation BTS (Brazilian Tensile Strength) should better be specified also in the caption or in the axis title.

7. Figure 2: The two decimal digits are not needed here.

8. Introduction, Line 34: Please consider "The present article is assessing the impact of rapid cooling (as compared to air cooling) on ...” or similar.

9. Method, Line 37: Please specify how many samples were subjected to each of the three tests.

10. Please consider adding a schematic illustration of the experimental setup and the various processes.

11. Line 39: The power of $15 \mathrm{~kW}$ was probably the nominal generator power, which is not necessarily the effective power absorbed by the sample. Please indicate the reflected power (if available) or add a note regrading the actual microwave-power definition.

12. Results: Please present the temperature evolution cycle (preferably by a temperature vs. time graph) or at least indicate the maximal and minimal temperatures attained in the microwave-heating and the cooling stages. The quantitative values of the various cooling rates are also essential here. 
Is the data presented in the most useful manner? (40\%) 4/5

Does the paper cite relevant and related articles appropriately? (30\%) 5/5

Context

Does the abstract correctly embody the content of the article? (25\%)

Does the introduction give appropriate context? (25\%)

Is the objective of the experiment clearly defined? (25\%)

Analysis

Are the limitations of the experiment as well as the contributions of the experiment clearly outlined? $(20 \%)$ 